\title{
Identification of the miRNA-target gene regulatory network in intracranial aneurysm based on microarray expression data
}

\author{
KEZHEN WANG ${ }^{1 *}$, XINMIN WANG ${ }^{1 *}$, HONGZHU LV $^{1 *}$, CHENGZHI CUI $^{1 *}$, JIYONG LENG $^{1}$, \\ KAI XU ${ }^{2}$, GUOSONG YU ${ }^{2}$, JIANWEI $\mathrm{CHEN}^{2}$ and PEIYU CONG ${ }^{1}$ \\ ${ }^{1}$ Department of Neurosurgery, Dalian Municipal Central Hospital Affiliated to \\ Dalian Medical University, Dalian, Liaoning 116033; ${ }^{2}$ Dalian Medical University Graduate \\ School, Dalian Medical University, Dalian, Liaoning 116044, P.R. China
}

Received December 10, 2015; Accepted January 26, 2017

DOI: $10.3892 / \mathrm{etm} .2017 .4378$

\begin{abstract}
Intracranial aneurysm (IA) remains one of the most devastating neurological conditions. However, the pathophysiology of IA formation and rupture still remains unclear. The purpose of the present study was to identify the crucial microRNA (miRNA/miR) and genes involved in IAs and elucidate the mechanisms underlying the development of IAs. In the present study, novel miRNA regulation activities in IAs were investigated through the integration of public gene expression data of miRNA and mRNA using the Gene Expression Omnibus database, combined with bioinformatics prediction. A total of 15 differentially expressed miRNA and 1,447 differentially expressed mRNA between IAs and controls were identified. A number of miRNA-target gene pairs (770), whose expression levels were inversely correlated, were used to construct a regulatory network of miRNA-target genes in IAs. The biological functions and pathways of these target genes were revealed to be associated with IAs. Specific miRNA and genes, such as hsa-let-7f, hsa-let-7d, hsa-miR-7, RPS6KA3, TSC1 and IGF1 may possess key roles in the development of IAs. The integrated analysis in the present study may provide insights into the understanding of underlying molecular mechanisms of IAs and novel therapeutic targets.
\end{abstract}

\section{Introduction}

Intracranial aneurysms (IAs), also referred to as cerebral aneurysms, are balloons or sac-like dilatations of arteries inside the brain. To date, IAs remain to be one of the most devastating

Correspondence to: Dr Peiyu Cong, Department of Neurosurgery, Dalian Municipal Central Hospital Affiliated to Dalian Medical University, 826, Xinan Road, Shahekou, Dalian, Liaoning 116033, P.R. China

E-mail: cong_peiyu@163.com

*Contributed equally

Key words: mRNA expression data, miRNA expression data, intracranial aneurysms, regulatory network, miRNA target genes neurological conditions with a prevalence of 2-3\% in the general population (1). Unruptured IAs are typically asymptomatic; however, in the event that IAs rupture, this process results in hemorrhage to the subarachnoid space, which is a devastating condition that has been indicated to have a mortality rate of $30-40$, and $50 \%$ of survivors are left disabled (2).

Previous research on the etiology of IAs indicated that the formation of IAs is assumed to be caused by diverse environmental and genetic factors, such as cigarette smoking, excessive alcohol consumption, hypertension, female gender and family history of IAs (3-5). However, the pathophysiology of IA formation and rupture still remains to be fully elucidated.

Microarray-based gene expression analyses have implied several mechanisms underlying the development of IAs (6-10). Extracellular matrix turnover factors and inflammatory factors, such as interleukin (IL)-1 $\beta$, IL-6, IL-8, IL-18, interferon- $\gamma$, tumor necrosis factor- $\alpha$ and major histocompatibility complex class II gene, have essential roles in the development, progression, and rupture of aneurysms $(11,12)$. Several pathways, including those associated with inflammatory responses, the immune system, extracellular matrix, and apoptosis are considered to be crucial in the formation, progression, and rupture of IAs $(8,13)$.

MicroRNA (miRNA/miR) are small, non-coding, single-stranded RNA, which are implicated in the post-transcriptional regulation of gene expression of either mRNA degradation or inhibiting translation, followed by protein synthesis repression (14). Furthermore, miRNA may modulate pathways and mechanisms of IAs via the control in gene expression. Previous studies have demonstrated that miRNA are involved in vascular remodeling and atherosclerosis $(15,16)$. In addition, a previous study revealed that a subset of inflammation-related miRNA were specifically upregulated in stroke patients with intracerebral hemorrhage and indicated that miR-16, and miR-25 were independent factors for IA occurrence by screening the miRNA expression level of 40 IA patients (20 unruptured and 20 ruptured) and 20 healthy volunteers via microarray assays and reverse transcription-quantitative polymerase chain reaction (RT-qPCR) analysis (17).

In the present study, bioinformatic methods were used to merge miRNA and mRNA expression data separately, using 
Table I. Characteristics of mRNA and miRNA expression profiling of IAs.

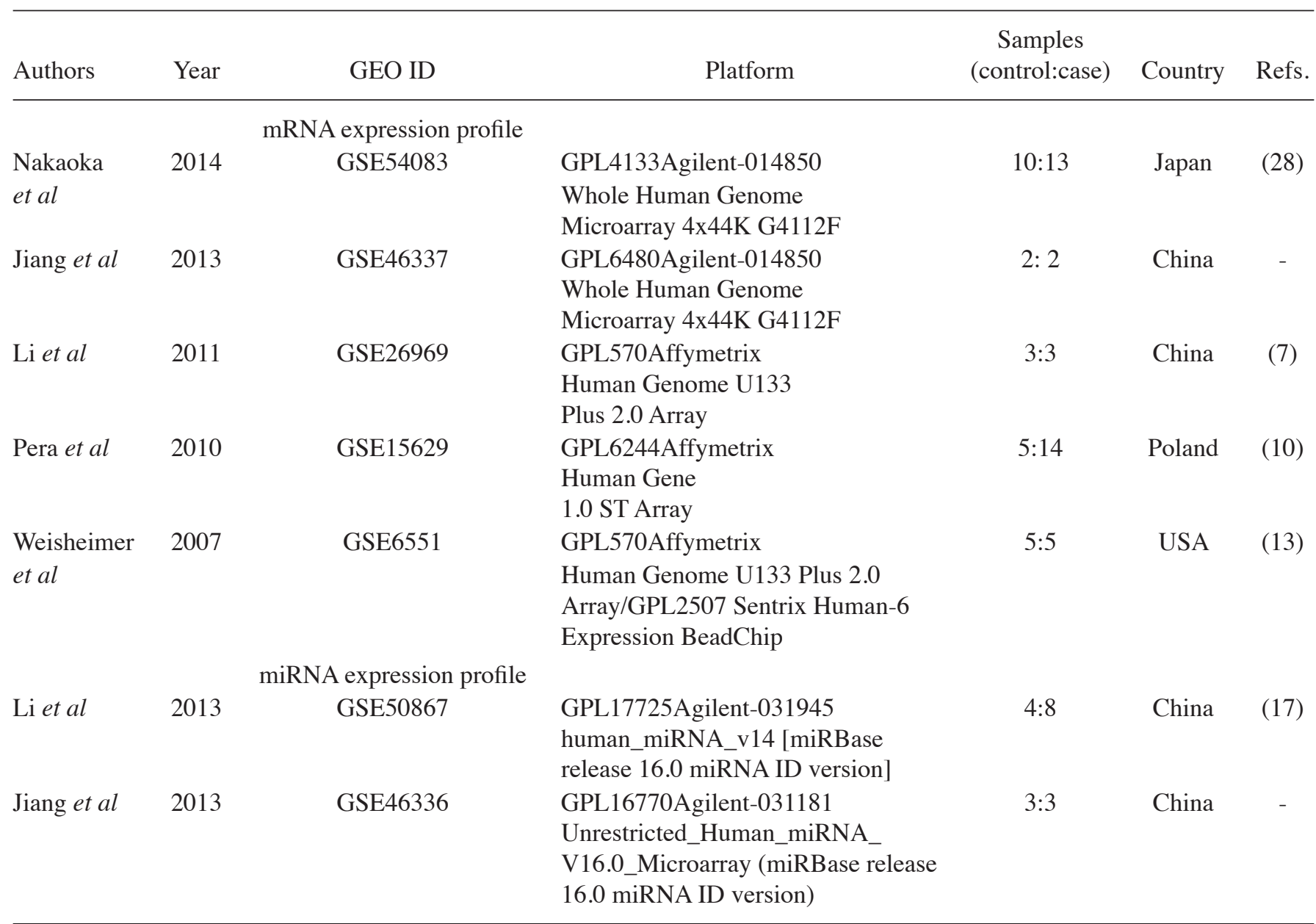

miRNA, microRNA; GEO, Gene Expression Omnibus database.

data available on the Gene Expression Omnibus database (GEO), to identify differentially expressed mRNA and miRNA between IAs and normal tissues. Subsequently, differentially expressed miRNA target genes were detected by bioinformatics prediction, inversely correlated analysis of miRNA and mRNA expressions were conducted and a miRNA-target gene regulatory network was constructed. The present findings may contribute to future investigations aimed at elucidaing the mechanisms of IAs.

\section{Materials and methods}

Eligible gene expression profiles. IA expression profiling studies were searched on the GEO database (ncbi.nlm.gov/geo), which serves as a public repository for gene expression datasets to meet the growing demand for a public repository for high-throughputgene expression data(18).IA expressionprofiling studies were only retained if they compared miRNA or mRNA expression profiling between IAs and normal tissues.

Differential analysis of miRNA and $m R N A$. Raw microarray data from each study was downloaded, and preprocessed with $\log _{2}$ transformation and Z-score normalization. The Linear Models for Microarray Data package in R (r-project. org) was used to identify the differently expressed probe sets between IAs and controls using the two-tailed Student's t-test and P-values of individual microarray studies were obtained. MetaMA package in R (r-project.org) was used to combine $\mathrm{P}$-values from multiple microarray studies and false discovery rate (FDR) was calculated for multiple comparisons using the Benjamini \& Hochberg method (19). We selected differently expressed mRNA with criterion of FDR $<0.01$ and a criterion of FDR $<0.01$ for differently expressed miRNA. Heat map analysis was performed using the 'heatmap.2' function of the R/Bioconductor package 'gplots' (20).

Identification of differently expressed miRNA target genes. To understand the potential association between differentially expressed mRNA and miRNA obtained in the present study, the transcriptional targets of the identified miRNA were predicted using the online tools of miRWalk (umm.uni-heidelberg. de/apps/zmf/mirwalk/) (21) based on six bioinformatic algorithms (DIANAmT, miRanda, miRDB, miRWalk, PICTAR and TargetScan). Putative targets that were common in the prediction of $\geq 4$ algorithms were selected to match with those identified to be dysregulated in IAs. As miRNA tend to decrease the expression of their target mRNA, differentially expressed target genes whose expression levels were inversely 
Table II. List of differentially expressed miRNA.

\begin{tabular}{lcc}
\hline miRNA & FDR & Fold-change \\
\hline $\begin{array}{l}\text { Upregulated miRNA } \\
\text { hsa-miR-188-5p }\end{array}$ & $2.79 \mathrm{E}-09$ & \\
hsa-miR-1183 & $9.74 \mathrm{E}-08$ & 2.9750 \\
hsa-miR-18a & $6.43 \mathrm{E}-06$ & 3.4515 \\
hsa-miR-7 & $5.18 \mathrm{E}-05$ & 2.6047 \\
hsa-miR-590-5p & $2.85 \mathrm{E}-04$ & 2.6007 \\
hsa-let-7d & $1.77 \mathrm{E}-03$ & 2.3965 \\
hsa-let-7f & $2.17 \mathrm{E}-03$ & 2.3005 \\
hsa-miR-130b & $7.24 \mathrm{E}-03$ & 2.3227 \\
hsa-miR-324-3p & $8.20 \mathrm{E}-03$ & 1.8100 \\
hsa-miR-1914a & $8.20 \mathrm{E}-03$ & 2.4691 \\
Downregulated miRNA & & \\
hsa-miR-425 & & -3.4574 \\
hsa-miR-182 & $1.98 \mathrm{E}-05$ & -1.3745 \\
hsa-miR-1825 & $5.39 \mathrm{E}-04$ & -3.1043 \\
hsa-miR-139-5p & $1.80 \mathrm{E}-03$ & -1.5437 \\
hsa-miR-193b & $2.17 \mathrm{E}-03$ & -1.3729 \\
\end{tabular}

aTarget predictions were not available via the miRWalk database. miRNA, microRNA; FDR, false discovery rate.

correlated with that of miRNA were to subjected to further investigation (22-24).

Functional annotation. Functional enrichment analysis is essential to uncover biological functions of miRNA target genes. To gain insights into the biological functions of miRNA target genes, Gene Ontology (GO) classification and Kyoto Encyclopedia of Genes and Genomes (KEGG) pathway enrichment analysis was performed using GENECODIS online software (genecodis.cnb.csic.es) (25). GO, which includes three categories (biological process, molecular function and cellular component), provides a common descriptive framework of gene annotation and classification for analyzing gene set data. KEGG pathway enrichment analysis was also performed to detect the potential pathway of miRNA target genes based on the KEGG pathway database, which is a recognized and comprehensive database including various types of biochemistry pathways (26). FDR $<0.05$ was set as the cut-off for selecting significantly enriched functional GO terms and KEGG pathway.

Construction of the regulatory network of miRNA-target gene in IA. miRNA-target gene interaction networks in IA with miRNA-target gene interacting pairs with expression levels that were inversely correlated were investigated. miRNA regulation networks were visualized using Cytoscape software (27).

\section{Results}

Differentially expressed miRNA and $m R N A$ in IA. In the present study, two miRNA and five mRNA expression profiling studies $(7,10,13,17,28)$ of IA were collected
(Table I). Following normalization of the original miRNA and mRNA expression datasets, 15 miRNA were regarded as significantly differentially expressed under the threshold of FDR $<0.01$. A total of 10 upregulated and five downregulated miRNA were identified (Table II). The upregulated miRNA with the lowest FDR was hsa-miR-188-5p, and the downregulated gene with the lowest FDR was hsa-miR-425. The significantly differentially expressed miRNA were displayed in a heat map (Fig. 1) and 1,447 genes were identified to be significantly differentially expressed between IAs and controls; 682 genes were upregulated and 765 genes were downregulated.

Regulatory network of miRNAs and target genes in IA. The miRWalk database was used to predict putative targets of significantly upregulated or downregulated miRNA in IAs. Comparing those putative targets with the list of differentially expressed genes in IAs, miRNA-target gene pairs with inversely correlated expression levels were selected. As a result, 531 miRNA-target gene pairs for the upregulated miRNA, with 29 pairs validated by experiments, and 211 miRNA-target gene pairs for the down regulated miRNA, with nine pairs validated by experiments, were identified (Table III). The target predictions of hsa-miR-1914 and hsa-miR-425 were not available in miRWalk databases.

Using the 770 miRNA-target gene pairs, a miRNA-target gene regulatory network was constructed. In the miRNA-target gene regulatory network, the top ten miRNA, which included hsa-miR-7, hsa-miR-182, hsa-miR-324-3p, hsa-miR-139-5p, hsa-miR-130b, hsa-let-7f, hsa-miR-18a, hsa-miR-188-5p, hsa-let-7d and hsa-miR-590-5p, were identified to regulate the greatest number of target genes, and the target genes, such as RPS6KA3, TSC1, AIM1, GAS7, GFOD1, GGA2, IGF1, IL28RA and INSR, were regulated by the greatest number of miRNA (Fig. 2).

GO classification and KEGG pathways of miRNA target genes. GO classification and KEGG pathway enrichment analysis were performed for miRNA target genes that were differently expressed. Peptide transport (GO, 0015833; FDR, 2.63E-01) and amide transport (GO, 0042886; FDR, 1.31E-01) were indicated to be significantly enriched for biological processes. Molecular functions, ATP binding (GO, 0005524; FDR, 8.93E-02) and adenyl ribonucleotide binding (GO, 0032559; FDR, 6.09E-02) were also significantly enriched. Furthermore, cellular component, collagen trimer (GO, 0005581; FDR, 1.29E-02) and endoplasmic reticulum lumen (GO, 0005788; FDR, 1.30E-01) were significantly enriched (Table IV). The most significant pathway in the present KEGG analysis was focal adhesion (FDR, 1.07E-08). Pathways in cancer (FDR, 2.49E-08) and cytokine-cytokine receptor interaction $(\mathrm{P}=5.88 \mathrm{E}-08)$ were also indicated to be highly enriched (Table V).

\section{Discussion}

IAs are considered to be the most fatal cerebrovascular system disease and are characterized by the apoptosis of smooth muscle cells, degeneration of vessel walls, and activation of 
Table III. miRNA-mRNA pairs with inversely correlated expression levels.

\begin{tabular}{|c|c|c|c|}
\hline miRNA & $\begin{array}{c}\text { Regulation } \\
\text { (miRNA) }\end{array}$ & $\begin{array}{l}\text { Target } \\
\text { counts }\end{array}$ & Target mRNA \\
\hline
\end{tabular}

\begin{tabular}{lll}
\hline hsa-miR-188-5p Up & AEBP2, ATP6V1G1, BAG5, BCL9, BET1, BIN2, CAPN2, CD80, CDC25B, \\
& CDON, CLU, CPSF2, CYP1A1, CYYR1, DAAM1, DLC1, FBXO11, FBXO9, \\
& FNBP1, FNBP4, FUBP3, GL1, HMGB1, IL28RA, IL2RA, ING5, KPTN, MX2, \\
& MYT1, N6AMT1, PAX8, PCDH9, PER2, PLVAP, PROS1, PSMF1, RPS6KA3, \\
& SCN3A, SH3BGR2, SLC22A3, SPG20, SPOP, SYNJ2BP, SYT11, SYTL2, \\
& TACC1, TCL1A, TIAM1, TSN, UPF2, UPF3A, USP14, USP47, UVRAG, \\
& ZNF185, ZNF451, ITLN1, GEMIN4, PCNA
\end{tabular}

$\begin{array}{llc}\text { hsa-miR-1183 } & \text { Up } & 9 \\ \text { hsa-miR-18a } & \text { Up } & 63\end{array}$

AEBP2, AIM1, ARG2, CRIM1, DARS, F8, POT1, ROCK2, SLC9A6

hsa-miR-18a Up $\quad 63$

TP53, GEMIN4, CD8A, ADCY1,AEBP2,AIM1, C1RL, C9orf5, CBX7, CLOCK, CNTN4, CRELD1, CRIM1, CTLA4, DAAM2, DUSP3, EFS, EGLN2,

EYA4, FBXO9, FNBP1, GAS7, GFOD1, IGF1, IL28RA, INSR, ITIH5, KIF3B, MC2R, MS4A2, NDUFS1, NEDD4, NPY1R, OSTM1, PCNP, PCSK2, PLEKHG1, POT1, POU6F1, PRMT6, PSMF1, RERG, RPS6KA3, RRAS, SH3BGRL2, SH3BP4, SLC22A3, SLC6A7, SON, SOX8, SRI, STARD7, STAT6, TSC1, TXNIP, UQCRB, USP24, VPS4B, WASF2, ZHX2, ZNF169, ZNF430, ZNF451

hsa-miR-7

hsa-miR-590-5p

Up

49

hsa-let-7d

Up

55

hsa-let-7f

Up

68

hsa-miR-130b
94

AIM1, ARID2, ATP6V1G1, BAG5, C1GALT1, CAMK1G, CAMK2D, CAPN2, CBX7, CD5, CD8A, CDC14B, CDON, CISH, CLNS1A, COLEC12, CRIM1, CRY2, CXCL12, DCK, DMXL1, EVC, FAM20B, FBLN5, FBXO21, GAS7, GATA4, GATA6, GGA2, GNG4, GRIK3, HN1, IGF1, INHBB, INSR, ITIH5, JAM3, KIF3B, LDB3, LGALS8, LIFR, LUC7L2, MAOA, MC2R, MEIS2, MFAP4, MIPOL1, MRPS36, N6AMT1, NEDD4, NEIL1, PARD6G, PAX8, PIGH, PIK3R3, POLE3, POU6F1, PPP2R2B, PPP2R3A, ProSAPiP1, PSORS1C2, PUM2, RAB5B, RAP1A, RFC5, RIMS3, ROCK2, RPS6KA3, SEMA6A, SLC22A3, SLC9A6, SNAP29, SQSTM1, SS18L1, STEAP2, SYNJ2BP, SYNPR, TCERG1, TCL1A, TGFA, THAP6, TIAM1, TMOD1, TMOD2, TOX, TRDN, TRIM52, TRPV1, TSC1, TSN, UTRN, ZFYVE21, ZNF185, ZNF319

ANXA1, BBS7, C14orf101, CAMK2D, CAPN2, CNTFR, COL4A4, CRIM1, CTCF, CUBN, DNAJA2, DNM1L, ENPP4, FAT3, FBXO11, FUBP3, FZD6, GFOD1, INSR, IRAK1BP1, ITIH5, KIAA0240, KL, KLF8, LIFR, MAOA, MATN2, MS4A2, OLFM3, PCNA, PELI1, PER2, POT1, PPP1R3D, RABIF, REPS1, RIOK1, RNF38, RPS6KA3, SLMAP, SMARCE1, SRI, TIAM1, TPK1, TRUB1, TSC1, USP24, WSB1, ZNF295

TAF9, CLU, IGF1, XPO1, TP53, ACTA2, CISH, IFNG, FMR1, ABCB9, ADCY9, AIM1, C14orf28, C1QTNF1, C1RL, CALM1, CD80, CDC14B, CDC25B, CDCA8, COL14A1, COL4A4, CRY2, DPF2, DUSP19, ENPP4, FRAS1, GAS7, GFOD1, GGA2, GNG5, ICOS, IL28RA, INSR, KIAA1609, LDB3, LEPROTL1, LIFR, MC2R, MTHFD2, MUC4, P2RX1, PRDM12, PSORS1C2, ROBO4, RPS6KA3, SCARA3, SPOP, SYT11, TNFSF10, TPK1, TRIM39, TSC1, USP24, UTRN

ABCB9, ADCY1, ADCY9, AIM1, ATP6V1G1, BMPR1A, C14orf28, C1QTNF1, C1RL, CALM1, CD80, CD8A, CDC14B, CDC25B, CDCA8, CISH, CLU, COL14A1, COL4A4, COL4A5, CRY2, DIABLO, DLC1, DPF2, DUSP19, EGLN2, ENPP4, FMR1, FRAS1, GALR1, GAS7, GFOD1, GGA2, GNG5, ICOS, IFNG, IGF1, IL28RA, IL2RA, INSR, KIAA1609, LDB3, LEPROTL1, MC2R, MEIS2, MTHFD2, MUC4, NTRK3, P2RX1, PAX8, PSORS1C2, RIMS3, RNF38, ROBO4, RPS6KA3, SCARA3, SIPA1L2, SPOP, SYNJ2BP, SYT11, TNFSF10, TP53, TPK1, TSC1, USP24, USP47, UTRN, XPO1

ADCY1, ADD2, AIG1, ANK2, BAI3, C1GALT1, CALM2, CAMK2D, CCR6, CDON, CLOCK, CNTN4, COL4A4, CRY1, CXCL12, DEDD2, DNM1L, DOK5, DUSP19, EFS, ENPP4, EPHB4, FAM20B, FAT3, FBXO9, FMR1, FNBP1, FZD6, GGA2, HOXA3, IGF1, IL28RA, INHBB, ITPR1, LDB3, LEPROTL1, METAP1, MLLT10, MRRF, MTMR4, MYT1, NEIL1, PCYOX1, PELI1, PLSCR4, POU6F1, PPIA, PPP1R12A, PTPRG, RAB5B, RNF38, RPS6KA3, 
Table III. Continued.

\begin{tabular}{|c|c|c|}
\hline miRNA & $\begin{array}{l}\text { Regulation } \\
\text { (miRNA) }\end{array}$ & $\begin{array}{l}\text { Target } \\
\text { counts }\end{array}$ \\
\hline
\end{tabular}

\begin{tabular}{|c|c|c|c|}
\hline & & & $\begin{array}{l}\text { RYR2, SCARA3, SCN3A, SLC9A6, SLMAP, SOX21, SPG20, SRPX, STOM, } \\
\text { TACC1, TBC1D8, TGFA, THAP6, TSC1, TXNIP, USP47, VPS4B, WDR1, } \\
\text { WRN, ZAK, ZNF430 }\end{array}$ \\
\hline hsa-miR-130b & Up & 73 & $\begin{array}{l}\text { ADCY1, ADD2, AIG1, ANK2, BAI3, C1GALT1, CALM2, CAMK2D, CCR6, } \\
\text { CDON, CLOCK, CNTN4, COL4A4, CRY1, CXCL12, DEDD2, DNM1L, DOK5, } \\
\text { DUSP19, EFS, ENPP4, EPHB4, FAM20B, FAT3, FBXO9, FMR1, FNBP1, } \\
\text { FZD6, GGA2, HOXA3, IGF1, IL28RA, INHBB, ITPR1, LDB3, LEPROTL1, } \\
\text { METAP1, MLLT10, MRRF, MTMR4, MYT1, NEIL1, PCYOX1, PELI1, } \\
\text { PLSCR4, POU6F1, PPIA, PPP1R12A, PTPRG, RAB5B, RNF38, RPS6KA3, } \\
\text { RYR2, SCARA3, SCN3A, SLC9A6, SLMAP, SOX21, SPG20, SRPX, STOM, } \\
\text { TACC1, TBC1D8, TGFA, THAP6, TSC1, TXNIP, USP47, VPS4B, WDR1, } \\
\text { WRN, ZAK, ZNF430 }\end{array}$ \\
\hline hsa-miR-324-3p & Up & 83 & $\begin{array}{l}\text { ADAMTS17, ADCY1, ANKRD11, ARHGAP10, ARHGEF17, BARHL1, BRD2, } \\
\text { C1QTNF1, CAMK1G, CBX7, CD34, CD5, CD8A, CDC14B, CDC25B, CLU, } \\
\text { COL14A1, COL21A1, CRY2, CXCL12, CYGB, DIABLO, DLC1, DNAJB2, } \\
\text { EFS, EGLN2, ELTD1, EPHB4, ESAM, FBXO9, FNBP1, GAS7, GATA4, } \\
\text { GFOD1, GGA2, GRIK3, HOXD4, KCND1, KCNS1, KIAA1609, KIF3B, } \\
\text { LEPROTL1, LGALS8, LUC7L2, MYLK2, MYT1, NGFR, NTRK3, NUDC, } \\
\text { PAX8, PIK3R3, PODN, PPAP2B, PPP1R3D, PRKAR1A, ProSAPiP1, PSMF1, } \\
\text { PSORS1C2, RAB5B, RARG, RBM3, RGS3, RGS6, RIMS3, RPL13A, } \\
\text { RPS6KA3, SLAMF7, SLC6A7, SOX21, SOX8, SS18, SYT11, TRPV3, TSC1, } \\
\text { USP22, USP47, UVRAG, VASP, WASF2, YWHAG, ZNF319, ZNF451, ZNF510 }\end{array}$ \\
\hline hsa-miR-182 & Down & 84 & $\begin{array}{l}\text { CDKN1A, MYCN, BAX, EGR1, DOK4, MBNL2, ARF4, PCDH8, XPR1, } \\
\text { DDAH1, GALNT2, KITLG, PAPPA, KCNK10, SLC2A3, THBS1, THBS2, } \\
\text { ZIC3, GPR68, HOOK3, ADAM9, NAV1, LHFPL2, SIRPB1, VAT1, YKT6, } \\
\text { KHDRBS3, MAL2, SLC36A4, COL5A1, SLC31A1, ASB6, CYBB, RDH10, } \\
\text { DDX3X, DLAT, FBLN1, C6orf89, MRAS, NLGN4Y, LPHN1, MESDC2, } \\
\text { SYNE2, FN1, KIAA0368, NUDT13, D4S234E, KCNH5, HTR2C, KIAA2022, } \\
\text { IL16, KIF5C, LRP1, MCL1, MKLN1, OLR1, PC, CECR1, WHSC1L1, PCDHB4, } \\
\text { SH3GLB2, THAP10, USP31, PTPRE, NTN4, RNASE6, RNASEL, RRBP1, } \\
\text { SCNN1G, SLC11A1, SLC22A5, SRPK1, SSTR2, TBL1X, SLC35A2, TNFSF9, } \\
\text { CCND2, NAV2, ACVR1B, SYNGR2, GCM2, KCNK6, KIF23, KIAA0247 }\end{array}$ \\
\hline hsa-miR-1825 & Down & 6 & CDH2, ABCA1, PANX1, SERPINE1, NLK, C2orf3 \\
\hline hsa-miR-139-5p & Down & 80 & $\begin{array}{l}\text { MBNL2, DDX3X, ABCA1, FOS, GALNT3, ENAH, TBL1X, PTPRU, DPYSL4, } \\
\text { YKT6, ABHD2, BAZ2A, SLC6A14, SLC35A4, MAL2, AP2M1, CCR5, } \\
\text { COL11A1, CX3CR1, RDH10, ARX, DSC2, EFNA3, EREG, C6orf89, MRAS, } \\
\text { FN1, DDAH1, NR5A1, GALNT2, D4S234E, GLI3, GNAL, USP25, NME7, } \\
\text { EHD4, HOXA7, KIAA2022, IGFBP5, HCN1, IL16, JAK3, KCNA3, KIF5C, } \\
\text { LHCGR, MARK1, MBNL1, MCL1, KITLG, NF2, PPAT, DIRAS2, BCAS3, } \\
\text { PPP2R4, DOK4, PRKCA, CYP4F11, RFXAP, RNASEL, RPL15, SCD, SMOC1, } \\
\text { SLC39A8, SGCD, SRPK1, TGFB1, THBS1, TPM3, UFD1L, CUL3, PPFIBP1, } \\
\text { KIAA1755, STX11, TNFRSF10D, CCND2, SLC28A2, GCM2, TM9SF4, } \\
\text { SPOCK2, TAGLN2 }\end{array}$ \\
\hline hsa-miR-193b & Down & 47 & $\begin{array}{l}\text { SOX9, MCL1, BCL2L10, STMN1, MKLN1, PTPRU, ABHD2, BAZ2A, AP2M1, } \\
\text { SLC31A1, CRK, CX3CR1, E2F1, DDAH1, KCNE1L, GALNT2, TNFRSF21, } \\
\text { GCLC, APOA2, IGFBP5, MMP14, MYCN, NF2, NUMA1, SERPINE1, } \\
\text { RNF141, PPAT, DIRAS2, PPP2R4, PRKCA, KIAA1199, PTPRE, SGCD, } \\
\text { SLC20A2, SOX12, TRAF1, ZIC3, AXIN2, HAVCR2, KIAA1755, TNFRSF10B, } \\
\text { CCND2, NAV1, SOCS3, ACVRL1, BCAR1, KIAA0195 }\end{array}$ \\
\hline
\end{tabular}

the immune system in the aortic wall (29). miRNA have been revealed to be critical modulators in vascular biology and disease, such as atherosclerosis, arterial remodeling, angiogenesis, and smooth muscle cell regeneration (30). Additionally, 


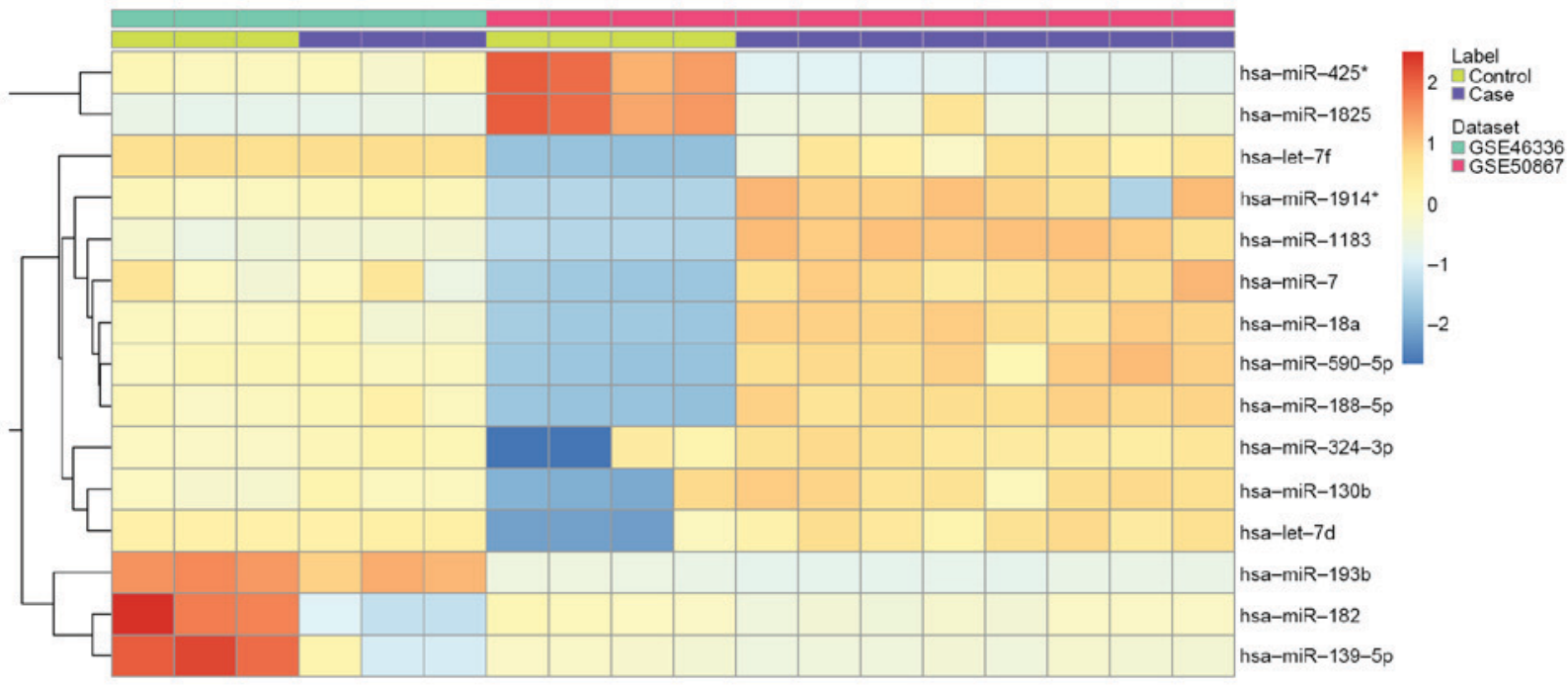

Figure 1. Heat-map image representing 15 miRNA that were significantly upregulated or downregulated (false discovery rate $<0.01$ ) in intracranial aneurysms compared with normal controls.
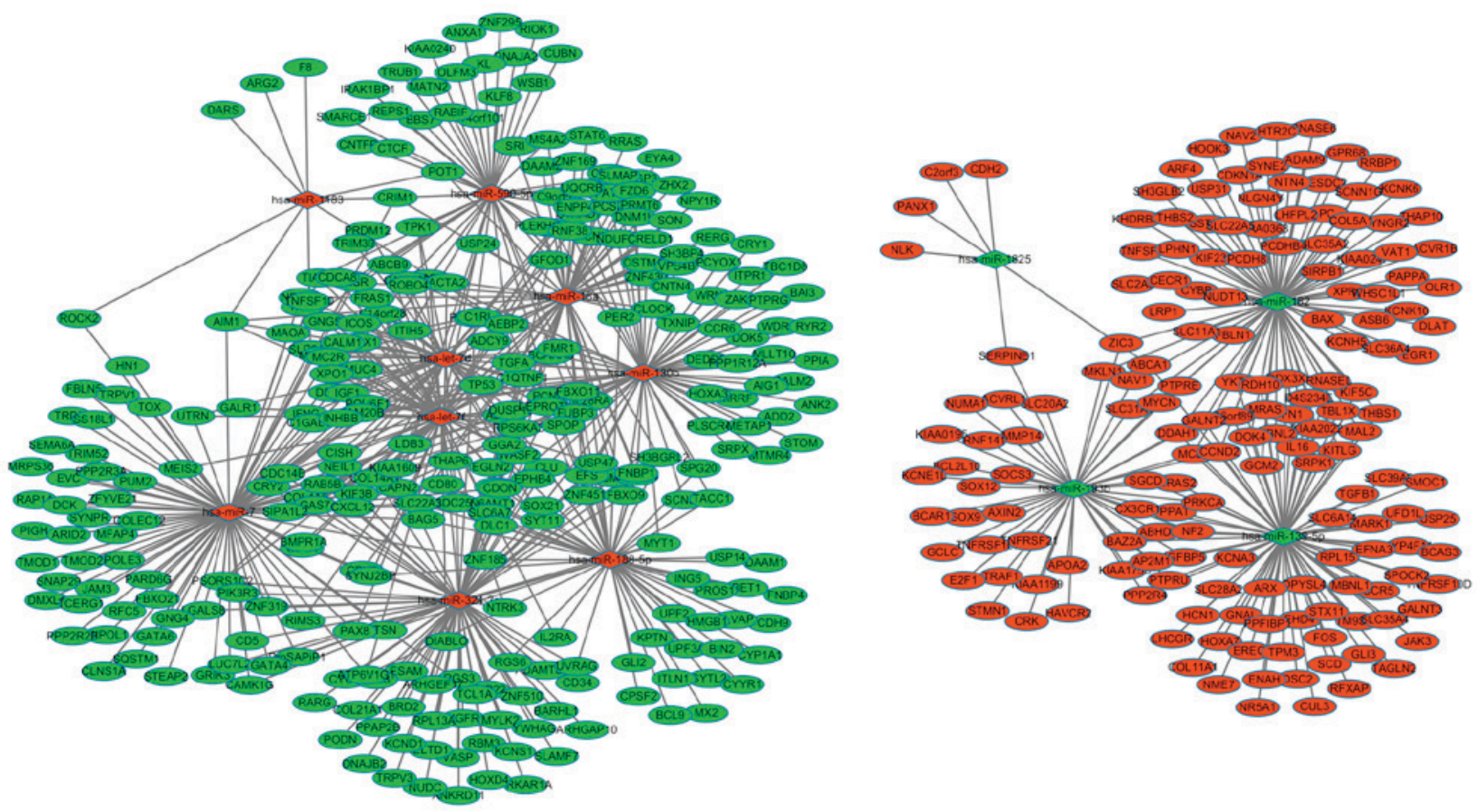

Figure 2. Regulatory network between miRNA and target genes in intracranial aneurysms. Diamonds and ellipses represent miRNA and genes, respectively. Red and green colors represent the relatively high and low expression, respectively. miRNA, microRNA.

miRNA may have vital roles in IA development by regulating downstream genes.

Studies have examined the miRNA and mRNA expression profiles in IA to identify differentially-expressed miRNA and genes. However, inconsistent results were obtained due to platform differences, tissue sampling and control selection in gene expression profiles $(9,31-33)$. In the present study, miRNA and mRNA expression data were integrated to identify differentially expressed miRNA and mRNA between IAs and normal tissues. Subsequently, 770 miRNA-target gene pairs with inversely correlated expression levels were identified via bioinformatics prediction were selected to construct a miRNA-target gene regulatory network in IA.
In the miRNA-target gene regulatory network, the top ten miRNA (hsa-miR-7, hsa-miR-182, hsa-miR-324-3p, hsa-miR-139-5p, hsa-miR-130b, hsa-let-7f, hsa-miR-18a, hsa-miR-188-5p, hsa-let-7d and hsa-miR-590-5p) were identified to regulate the greatest number of target genes. Target genes, such as RPS6KA3, TSC1, AIM1, GAS7, GFOD1, GGA2, IGF1, IL28RA and INSR, were indicated to be regulated by the greatest number of miRNA. hsa-let-7d and hsa-let-7f, two members of the let family, which are enriched in endothelium, were revealed to be differently expressed between six intracranial aneurysmal samples and normal superficial temporal arteries by genome-wide microRNA screening (32). hsa-miR-7, which is brain-enriched, may be 
Table IV. Top 15 GO functional annotations of differentially expression miRNA target genes.

\begin{tabular}{|c|c|c|c|c|}
\hline GO ID & GO term & Count & P-value & FDR \\
\hline \multicolumn{5}{|c|}{ Biological process } \\
\hline GO:0015833 & Peptide transport & 2 & $5.21 \mathrm{E}-05$ & 2.63E-01 \\
\hline GO:0042886 & Amide transport & 2 & $5.21 \mathrm{E}-05$ & $1.31 \mathrm{E}-01$ \\
\hline GO:0042327 & Positive regulation of phosphorylation & 19 & $1.13 \mathrm{E}-04$ & $1.91 \mathrm{E}-01$ \\
\hline GO:0044712 & Single-organism catabolic process & 15 & $2.38 \mathrm{E}-04$ & $3.00 \mathrm{E}-01$ \\
\hline GO:0010562 & $\begin{array}{l}\text { Positive regulation of phosphorus } \\
\text { metabolic process }\end{array}$ & 20 & $2.38 \mathrm{E}-04$ & $2.40 \mathrm{E}-01$ \\
\hline GO:0045937 & $\begin{array}{l}\text { Positive regulation of phosphate } \\
\text { metabolic process }\end{array}$ & 20 & $2.38 \mathrm{E}-04$ & $2.00 \mathrm{E}-01$ \\
\hline GO:0044763 & Single-organism cellular process & 320 & 2.73E-04 & $1.97 \mathrm{E}-01$ \\
\hline GO:0060191 & Regulation of lipase activity & 5 & $3.88 \mathrm{E}-04$ & 2.45E-01 \\
\hline GO:0030574 & Collagen catabolic process & 6 & $5.20 \mathrm{E}-04$ & 2.92E-01 \\
\hline GO:0032963 & Collagen metabolic process & 6 & $5.20 \mathrm{E}-04$ & 2.62E-01 \\
\hline GO:0044259 & $\begin{array}{l}\text { Multicellular organismal macromolecule } \\
\text { metabolic process }\end{array}$ & 6 & $5.20 \mathrm{E}-04$ & 2.39E-01 \\
\hline GO:0044243 & Multicellular organismal catabolic process & 6 & $5.20 \mathrm{E}-04$ & 2.19E-01 \\
\hline GO:0044236 & Multicellular organismal metabolic process & 6 & $5.20 \mathrm{E}-04$ & 2.02E-01 \\
\hline GO:0042325 & Regulation of phosphorylation & 20 & $6.27 \mathrm{E}-04$ & $2.26 \mathrm{E}-01$ \\
\hline GO:0032924 & Activin receptor signaling pathway & 2 & $7.48 \mathrm{E}-04$ & $2.52 \mathrm{E}-01$ \\
\hline \multicolumn{5}{|c|}{ Cellular component } \\
\hline GO:0005581 & Collagen trimer & 8 & $2.31 \mathrm{E}-05$ & $1.29 \mathrm{E}-02$ \\
\hline GO:0005788 & Endoplasmic reticulum lumen & 7 & $4.65 \mathrm{E}-04$ & $1.30 \mathrm{E}-01$ \\
\hline \multicolumn{5}{|c|}{ Molecular function } \\
\hline GO:0005524 & ATP binding & 7 & $9.26 \mathrm{E}-05$ & 8.93E-02 \\
\hline GO:0032559 & Adenyl ribonucleotide binding & 7 & $1.26 \mathrm{E}-04$ & 6.09E-02 \\
\hline GO:0030554 & Adenyl nucleotide binding & 7 & $1.26 \mathrm{E}-04$ & $4.06 \mathrm{E}-02$ \\
\hline GO:0043492 & $\begin{array}{l}\text { ATPase activity, coupled to movement } \\
\text { of substances }\end{array}$ & 2 & $1.47 \mathrm{E}-04$ & $3.53 \mathrm{E}-02$ \\
\hline GO:0015399 & Primary active transmembrane transporter activity & 2 & $1.47 \mathrm{E}-04$ & 2.83E-02 \\
\hline GO:0015405 & $\begin{array}{l}\text { P-P-bond-hydrolysis-driven transmembrane } \\
\text { transporter activity }\end{array}$ & 2 & $1.47 \mathrm{E}-04$ & $2.36 \mathrm{E}-02$ \\
\hline GO:0016820 & $\begin{array}{l}\text { Hydrolase activity, acting on acid anhydrides, } \\
\text { catalyzing transmembrane movement of substances }\end{array}$ & 2 & $1.47 \mathrm{E}-04$ & $2.02 \mathrm{E}-02$ \\
\hline GO:0042626 & $\begin{array}{l}\text { ATPase activity, coupled to transmembrane } \\
\text { movement of substances }\end{array}$ & 2 & $1.47 \mathrm{E}-04$ & $1.77 \mathrm{E}-02$ \\
\hline GO:0048185 & Activin binding & 2 & 2.69E-04 & 2.88E-02 \\
\hline GO:0016361 & Activin receptor activity, type I & 2 & 2.69E-04 & 2.59E-02 \\
\hline GO:0017002 & Activin-activated receptor activity & 2 & 2.69E-04 & 2.36E-02 \\
\hline GO:0051117 & ATPase binding & 3 & $3.01 \mathrm{E}-04$ & 2.42E-02 \\
\hline GO:0019901 & Protein kinase binding & 12 & $3.39 \mathrm{E}-04$ & $2.51 \mathrm{E}-02$ \\
\hline GO:0032549 & Ribonucleoside binding & 7 & $4.08 \mathrm{E}-04$ & 2.81E-02 \\
\hline GO:0032550 & Purine ribonucleoside binding & 7 & $4.08 \mathrm{E}-04$ & 2.62E-02 \\
\hline
\end{tabular}

GO, gene otology; FDR, false discovery rate; ATP, adenosine triphosphate.

implicated in the pathogenesis of glioblastoma, characterized by microvascular proliferation (34-36). Furthermore, hsa-miR-7 may function as a tumor suppressor gene to regulate glioblastoma microvascular endothelial cell proliferation by targeting RAF1. To the best of our knowledge, no reports of hsa-miR-7 in IAs have been published. In the present study, 94 targets of hsa-miR-7 were indicated to be significantly enriched in the mTOR signaling pathway and may modulate the apoptosis of muscle cell differentiation in IAs.

RPS6KA3, the target gene regulated by the greatest number of miRNA, has been indicated to be expressed 
Table V. KEGG pathway enrichment analysis of differentially expressed microRNA target genes (Top 15).

\begin{tabular}{|c|c|c|c|c|}
\hline KEGG ID & KEGG term & Count & FDR & Genes \\
\hline hsa04510 & Focal adhesion & 19 & $1.07 \mathrm{E}-08$ & $\begin{array}{l}\text { COL4A4, IGF1, PPP1R12A, ROCK2, CCND2, } \\
\text { BCAR1, COL4A5, RAP1A, THBS1, MYLK2, PRKCA, } \\
\text { CRK, PIK3R3, CAPN2, THBS2, VASP, COL5A1, } \\
\text { COL11A1, FN1 }\end{array}$ \\
\hline hsa05200 & Pathways in cancer & 23 & 2.49E-08 & $\begin{array}{l}\text { COL4A4, AXIN2, KITLG, FOS, FZD6, TGFA, IGF1, } \\
\text { GLI3, COL4A5, E2F1, TPM3, PRKCA, CRK, PIK3R3, } \\
\text { CDKN1A, TGFB1, BAX, GLI2, TP53, PAX8, TRAF1, } \\
\text { FN1, EGLN2 }\end{array}$ \\
\hline hsa04060 & $\begin{array}{l}\text { Cytokine-cytokine } \\
\text { receptor interaction }\end{array}$ & 20 & $5.88 \mathrm{E}-08$ & $\begin{array}{l}\text { INHBB, TNFRSF10D, KITLG, BMPR1A, TNFRSF10B, } \\
\text { CNTFR, IL28RA, TNFRSF21, CX3CR1, IFNG, LIFR, } \\
\text { TGFB1, IL2RA, NGFR, CCR5, ACVR1B, CXCL12, } \\
\text { TNFSF9, TNFSF10, CCR6 }\end{array}$ \\
\hline hsa05146 & Amoebiasis & 13 & $1.03 \mathrm{E}-07$ & $\begin{array}{l}\text { ARG2, COL4A4, GNAL, ADCY1, COL4A5, RAB5B, } \\
\text { IFNG, PRKCA, PIK3R3, TGFB1, COL5A1, COL11A1, } \\
\text { FN1 }\end{array}$ \\
\hline hsa04062 & $\begin{array}{l}\text { Chemokine } \\
\text { signaling pathway }\end{array}$ & 15 & $2.66 \mathrm{E}-06$ & $\begin{array}{l}\text { TIAM1, ADCY1, ROCK2, BCAR1, ADCY9, CX3CR1, } \\
\text { RAP1A, CRK, PIK3R3, JAK3, GNG5, CCR5, CXCL12, } \\
\text { GNG4, CCR6 }\end{array}$ \\
\hline hsa05214 & Glioma & 8 & $8.41 \mathrm{E}-05$ & $\begin{array}{l}\text { CAMK2D, TGFA, IGF1, E2F1, PRKCA, PIK3R3, } \\
\text { CDKN1A, TP53 }\end{array}$ \\
\hline hsa05144 & Malaria & 4 & $1.01 \mathrm{E}-04$ & IFNG, THBS1, TGFB1, THBS2 \\
\hline hsa04350 & $\begin{array}{l}\text { TGF-beta signaling } \\
\text { pathway }\end{array}$ & 4 & $1.01 \mathrm{E}-04$ & IFNG, THBS1, TGFB1, THBS2 \\
\hline hsa04670 & $\begin{array}{l}\text { Leukocyte } \\
\text { transendothelial } \\
\text { migration }\end{array}$ & 10 & $1.06 \mathrm{E}-04$ & $\begin{array}{l}\text { JAM3, ROCK2, BCAR1, ESAM, RAP1A, PRKCA, } \\
\text { PIK3R3, CYBB, VASP, CXCL12 }\end{array}$ \\
\hline hsa04115 & $\begin{array}{l}\text { p53 signaling } \\
\text { pathway }\end{array}$ & 8 & $1.18 \mathrm{E}-04$ & $\begin{array}{l}\text { TNFRSF10B, IGF1, CCND2, } \\
\text { THBS1, SERPINE1, CDKN1A, BAX, TP53 }\end{array}$ \\
\hline hsa04971 & $\begin{array}{l}\text { Gastric acid } \\
\text { secretion }\end{array}$ & 8 & $1.36 \mathrm{E}-04$ & $\begin{array}{l}\text { KCNK10, CAMK2D, ADCY1, ADCY9, MYLK2, } \\
\text { PRKCA, ITPR1, SSTR2 }\end{array}$ \\
\hline hsa04310 & $\begin{array}{l}\text { Wnt signaling } \\
\text { pathway }\end{array}$ & 11 & $1.42 \mathrm{E}-04$ & $\begin{array}{l}\text { NLK, CAMK2D, AXIN2, FZD6, ROCK2, CCND2, } \\
\text { PRKCA, DAAM2, TBL1X, TP53, DAAM1 }\end{array}$ \\
\hline hsa04722 & $\begin{array}{l}\text { Neurotrophin } \\
\text { signaling pathway }\end{array}$ & 10 & $1.56 \mathrm{E}-04$ & $\begin{array}{l}\text { CAMK2D, RAP1A, CRK, PIK3R3, YWHAG, NGFR, } \\
\text { BAX, RPS6KA3, TP53, NTRK3 }\end{array}$ \\
\hline hsa04630 & $\begin{array}{l}\text { Jak-STAT signaling } \\
\text { pathway }\end{array}$ & 11 & $1.58 \mathrm{E}-04$ & $\begin{array}{l}\text { CNTFR, CCND2, I L28RA, IFNG, LIFR, STAT6, } \\
\text { SOCS3, PIK3R3, JAK3, IL2RA, CISH }\end{array}$ \\
\hline hsa04020 & $\begin{array}{l}\text { Calcium signaling } \\
\text { pathway }\end{array}$ & 6 & $1.61 \mathrm{E}-04$ & $\begin{array}{l}\text { CAMK2D, ADCY1, ADCY9, MYLK2, PRKCA, } \\
\text { ITPR1 }\end{array}$ \\
\hline
\end{tabular}

KEGG, Kyoto Encyclopedia of Genes and Genomes; FDR, false discovery rate; TGF, transforming growth factor; STAT, signal transducer and activator of transcription.

in high levels in regions with high synaptic activity (37). Moreover, RPS6KA3 has been suggested to be associated with Coffin-Lowry syndrome, which causes severe mental problems sometimes associated with abnormalities of growth, cardiac abnormalities, kyphoscoliosis, as well as auditory and visual abnormalities (38). Molecular evidence from previous studies has revealed that RPS6KA3 may regulate neurotransmitter release by activating phospholipase $\mathrm{D}$ production of lipids required for exocytosis and that RPS6KA3 may also function as a proto-oncogene in multiple types of cancer targeted by corresponding miRNA $(39,40)$.
Mutations in either tuberous sclerosis (TSC) 1 or TSC2 suppressor genes are able to provoke tuberous sclerosis complex, which is an autosomal dominant disorder promoting the development of benign tumors in multiple organ systems, including the skin, brain, and kidneys, via increasing mammalian target of rapamycin (mTOR) activity $(41,42)$. TSC1 also has a role in arterial remodeling events by affecting the inflammatory and the growth-promoting response of angiotensin II (43). Insulin-like growth factor 1 (IGF1) expression has been indicated in the vasculature and lower IGF1 expression levels increased the risk of cardiovascular and abdominal 
aortic aneurysm in a previous study (44). Histological analysis in a swine aneurysm model has demonstrated that IGF1 is upregulated (4-fold) in thrombus organization (45).

With regards to the pathways that the identified target genes were involved in, focal adhesion was the most significant pathway revealed in KEGG analysis. This finding was consistent with a previous study by Shi et al (8), in which Illumina microarray analysis was performed on human the aneurysm wall of IAs. Based on the fact that IAs arise from progressive wall degeneration and remodeling in brain artery walls, focal adhesion may be involved in the pathogenesis of IA.

In conclusion, the present study identified 15 differentially expressed miRNA and 1,447 differentially expressed mRNA between IAs and normal tissues and constructed a regulatory network including 770 miRNA-target gene pairs with inversely correlated expression levels. In this network, several miRNA and genes that may possess key roles in IAs were discovered, such as miRNA hsa-let-7f, hsa-let-7d and hsa-miR-7, and genes, including RPS6KA3, TSC1 and IGF1. The biological pathway of focal adhesion may be involved in the pathogenesis of IA. The findings in the present study may contribute to future investigations aimed at elucidating the mechanisms of IAs.

\section{References}

1. Brown RD: Unruptured intracranial aneurysms. Semin Neurol 30: 537-544, 2010.

2. Nieuwkamp DJ, Setz LE, Algra A, Linn FH, de Rooij NK and Rinkel GJ: Changes in case fatality of aneurysmal subarachnoid haemorrhage over time, according to age, sex and region: A meta-analysis. Lancet Neurol 8: 635-642, 2009.

3. van Gijn J, Kerr RS and Rinkel GJ: Subarachnoid haemorrhage. Lancet 369: 306-318, 2007.

4. Tromp G, Weinsheimer S, Ronkainen A and Kuivaniemi $\mathrm{H}$ Molecular basis and genetic predisposition to intracranial aneurysm. Ann Med 46: 597-606, 2014.

5. Chalouhi N, Ali MS, Starke RM, Jabbour PM, Tjoumakaris SI, Gonzalez LF, Rosenwasser RH, Koch WJ and Dumont AS: Cigarette smoke and inflammation: Role in cerebral aneurysm formation and rupture. Mediators Inflamm 2012: 271582, 2012.

6. Krischek B, Kasuya H, Tajima A, Akagawa H, Sasaki T, Yoneyama T, Ujiie H, Kubo O, Bonin M, Takakura K, et al: Network-based gene expression analysis of intracranial aneurysm tissue reveals role of antigen presenting cells. Neuroscience 154: 1398-1407, 2008.

7. Li L, Yang X, Jiang F, Dusting GJ and Wu Z: Transcriptome-wide characterization of gene expression associated with unruptured intracranial aneurysms. Eur Neurol 62: 330-337, 2009.

8. Shi C, Awad IA, Jafari N, Lin S, Du P, Hage ZA, Shenkar R, Getch CC, Bredel M, Batjer HH and Bendok BR: Genomics of human intracranial aneurysm wall. Stroke 40: 1252-1261, 2009.

9. Marchese E, Vignati A, Albanese A, Nucci CG, Sabatino G, Tirpakova B, Lofrese G, Zelano G and Maira G: Comparative evaluation of genome-wide gene expression profiles in ruptured and unruptured human intracranial aneurysms. J Biol Regul Homeost Agents 24: 185-195, 2010.

10. Pera J, Korostynski M, Krzyszkowski T, Czopek J, Slowik A, Dziedzic T, Piechota M, Stachura K, Moskala M, Przewlocki R and Szczudlik A: Gene expression profiles in human ruptured and unruptured intracranial aneurysms: What is the role of inflammation? Stroke 41: 224-231, 2010.

11. Kao HW, Lee KW, Kuo CL, Huang CS, Tseng WM, Liu CS and Lin CP: Interleukin-6 as a prognostic biomarker in ruptured intracranial aneurysms. PLoS One 10: e0132115, 2015.

12. Sathyan S, Koshy LV, Srinivas L, Easwer HV, Premkumar S, Nair S, Bhattacharya RN, Alapatt JP and Banerjee M: Patho genesis of intracranial aneurysm is mediated by proinflammatory cytokine TNFA and IFNG and through stochastic regulation of IL10 and TGFB1 by comorbid factors. J Neuroinflammation 12: $135,2015$.
13. Weinsheimer S, Lenk GM, van der Voet M,Land S, Ronkainen A, Alafuzoff I, Kuivaniemi H and Tromp G: Integration of expression profiles and genetic mapping data to identify candidate genes in intracranial aneurysm. Physiol Genomics 32: 45-57, 2007.

14. Bartel DP: MicroRNAs: Genomics, biogenesis, mechanism and function. Cell 116: 281-297, 2004.

15. Small EM, Frost RJ and Olson EN: MicroRNAs add a new dimension to cardiovascular disease. Circulation 121: 1022-1032, 2010.

16. van Rooij E: The art of microRNA research. Circ Res 108: 219-234, 2011.

17. Li P, Zhang Q, Wu X, Yang X, Zhang Y, Li Y and Jiang F: Circulating microRNAs serve as novel biological markers for intracranial aneurysms. J Am Heart Assoc 3: e000972, 2014.

18. Edgar R, Domrachev M and Lash AE: Gene expression omnibus: NCBI gene expression and hybridization array data repository. Nucleic Acids Res 30: 207-210, 2002.

19. Reiner A, Yekutieli D and Benjamini Y: Identifying differentially expressed genes using false discovery rate controlling procedures. Bioinformatics 19: 368-375, 2003.

20. Reimers M and Carey VJ: Bioconductor: An open source framework for bioinformatics and computational biology. Methods Enzymol 411: 119-134, 2006.

21. Dweep H, Sticht C, Pandey P and Gretz N: MiRWalk-database: Prediction of possible miRNA binding sites by 'walking' the genes of three genomes. J Biomed Inform 44: 839-847, 2011.

22. Luo Z, Zhang L, Li Z, Li X, Li G, Yu H, Jiang C, Dai Y, Guo X, Xiang $\mathrm{J}$ and $\mathrm{Li} \mathrm{G}$ : An in silico analysis of dynamic changes in microRNA expression profiles in stepwise development of nasopharyngeal carcinoma. BMC Med Genomics 5: 3, 2012.

23. Lionetti M, Biasiolo M, Agnelli L, Todoerti K, Mosca L, Fabris S, Sales G, Deliliers GL, Bicciato S, Lombardi L, et al: Identification of microRNA expression patterns and definition of a microRNA/mRNA regulatory network in distinct molecular groups of multiple myeloma. Blood 114: e20-e26, 2009.

24. Enerly E, Steinfeld I, Kleivi K, Leivonen SK, Aure MR, Russnes HG, Rønneberg JA, Johnsen H, Navon R, Rødland E, et al: MiRNA-mRNA integrated analysis reveals roles for miRNAs in primary breast tumors. PLoS One 6: e16915, 2011.

25. Tabas-Madrid D, Nogales-Cadenas R and Pascual-Montano A: GeneCodis3: A non-redundant and modular enrichment analysis tool for functional genomics. Nucleic Acids Res 40 (Web Server issue): W478-W483, 2012.

26. Altermann E and Klaenhammer TR: Pathwayvoyager: Pathway mapping using the kyoto encyclopedia of genes and genomes (KEGG) database. BMC Genomics 6: 60, 2005.

27. Shannon P, Markiel A, Ozier O, Baliga NS, Wang JT, Ramage D, Amin N, Schwikowski B and Ideker T: Cytoscape: A software environment for integrated models of biomolecular interaction networks. Genome Res 13: 2498-2504, 2003.

28. Nakaoka H, Tajima A, Yoneyama T, Hosomichi K, Kasuya H, Mizutani T and Inoue I: Gene expression profiling reveals distinct molecular signatures associated with the rupture of intracranial aneurysm. Stroke 45: 2239-2245, 2014.

29. Brown RD Jr and Broderick JP: Unruptured intracranial aneurysms: Epidemiology, natural history, management options and familial screening. Lancet Neurol 13: 393-404, 2014.

30. Small EM and Olson EN: Pervasive roles of microRNAs in cardiovascular biology. Nature 469: 336-342, 2011.

31. Yu L, Fan J, Wang S, Zhang D, Wang R, Zhao Y and Zhao J: Gene expression profiles in intracranial aneurysms. Neurosci Bull 30: 99-106, 2014

32. Liu D, Han L, Wu X, Yang X, Zhang Q and Jiang F: Genome-wide microRNA changes in human intracranial aneurysms. BMC Neurol 14: 188, 2014.

33. Wei L, Gao YJ, Wei SP, Zhang YF, Zhang WF, Jiang JX, Sun ZY and $\mathrm{Xu} \mathrm{W}$ : Transcriptome network-based method to identify genes associated with unruptured intracranial aneurysms. Genet Mol Res 12: 3263-3273, 2013.

34. Liu Z, Liu Y, Li L, Xu Z, Bi B, Wang Y and Li JY: MiR-7-5p is frequently downregulated in glioblastoma microvasculature and inhibits vascular endothelial cell proliferation by targeting RAF1. Tumour Biol 35: 10177-10184, 2014.

35. Dong L, Li Y, Han C, Wang X, She L and Zhang H: MiRNA microarray reveals specific expression in the peripheral blood of glioblastoma patients. Int J Oncol 45: 746-756, 2014. 
36. Visani M, de Biase D, Marucci G, Cerasoli S, Nigrisoli E, Bacchi Reggiani ML, Albani F, Baruzzi A and Pession A; PERNO study group: Expression of 19 microRNAs in glioblastoma and comparison with other brain neoplasia of grades I-III. Mol Oncol 8: 417-430, 2014.

37. Zeniou M, Ding T, Trivier E and Hanauer A: Expression analysis of RSK gene family members: The RSK2 gene, mutated in coffin-lowry syndrome, is prominently expressed in brain structures essential for cognitive function and learning. Hum Mol Genet 11: 2929-2940, 2002.

38. Nishimoto HK, Ha K, Jones JR, Dwivedi A, Cho HM, Layman LC and Kim HG: The historical coffin-lowry syndrome family revisited: Identification of two novel mutations of RPS6KA3 in three male patients. Am J Med Genet A 164A: 2172-2179, 2014.

39. Polioudakis D, Abell NS and Iyer VR: MiR-191 regulates primary human fibroblast proliferation and directly targets multiple oncogenes. PLoS One 10: e0126535, 2015.

40. Song R, Liu Q, Hutvagner G, Nguyen H, Ramamohanarao K, Wong $\mathrm{L}$ and $\mathrm{Li}$ J: Rule discovery and distance separation to detect reliable miRNA biomarkers for the diagnosis of lung squamous cell carcinoma. BMC Genomics 15 (Suppl 9): S16, 2014.
41. Chan JA, Zhang H, Roberts PS, Jozwiak S, Wieslawa G, Lewin-Kowalik J, Kotulska K and Kwiatkowski DJ: Pathogenesis of tuberous sclerosis subependymal giant cell astrocytomas: Biallelic inactivation of TSC1 or TSC2 leads to mTOR activation. J Neuropathol Exp Neurol 63: 1236-1242, 2004.

42. Habib SL, Yadav A, Mahimainathan L and Valente AJ: Regulation of PI 3-K, PTEN, p53 and mTOR in malignant and benign tumors deficient in tuberin. Genes Cancer 2: 1051-1060, 2011.

43. Doyon P, van Zuylen WJ and Servant MJ: Role of IкB kinase- $\beta$ in the growth-promoting effects of angiotensin II in vitro and in vivo. Arterioscler Thromb Vasc Biol 33: 2850-2857, 2013.

44. Yeap BB, Chubb SA, McCaul KA, Flicker L, Ho KK, Golledge J, Hankey GJ and Norman PE: Associations of IGF1 and its binding proteins with abdominal aortic aneurysm and aortic diameter in older men. Eur J Endocrinol 166: 191-197, 2012.

45. Lee D, Yuki I, Murayama Y, Chiang A, Nishimura I, Vinters HV, Wang CJ, Nien YL, Ishil A, Wu BM and Viñuela F: Thrombus organization and healing in the swine experimental aneurysm model. Part I. A histological and molecular analysis. J Neurosurg 107: 94-108, 2007. 